(2)

\title{
New developments in the management of chronic lymphocytic leukemia: role of ofatumumab
}

This article was published in the following Dove Press journal:

OncoTargets and Therapy

20 January 2016

Number of times this article has been viewed

\author{
Luca Laurenti' \\ Idanna Innocenti' \\ Francesco Autore' \\ Simona Sica' \\ Dimitar G Efremov² \\ 'Department of Hematology, Catholic \\ University of the Sacred Heart, Rome, \\ ${ }^{2}$ Molecular Hematology, International \\ Centre for Genetic Engineering and \\ Biotechnology, Monterotondo, Italy
}

\begin{abstract}
Ofatumumab is one of the three anti-CD20 monoclonal antibodies currently available for the treatment of chronic lymphocytic leukemia (CLL). The US Food and Drug Administration (FDA) approved the use of ofatumumab in patients with CLL refractory to fludarabine and alemtuzumab in 2009, and the European Medicines Agency (EMA) granted approval for the same indication in 2010. Subsequent positive results of ofatumumab in combination with chlorambucil in treatment-naïve patients led the FDA in April 2014 to approve the use of this combination for first-line treatment of patients with CLL for whom fludarabine-based therapy is considered inappropriate. Later that year, the EMA approved the use of ofatumumab in combination with chlorambucil or bendamustine for the same indication. Ofatumumab has also shown potential as maintenance therapy for patients with relapsed CLL; an application to broaden the label for ofatumumab as maintenance therapy was submitted earlier this year to the EMA and FDA. Finally, ofatumumab has shown promising activity in combination with ibrutinib or idelalisib in relapsed/refractory CLL patients; combinations of ofatumumab with B-cell-receptor pathway inhibitors could represent another potential use of this antibody in the near future
\end{abstract}

Keywords: CLL, ofatumumab, monoclonal antibodies, immunotherapy

\section{Introduction}

Ofatumumab (HuMax-CD20, Arzerra ${ }^{\circledR}$; GlaxoSmithKline PLC, London, UK) is a recently approved, fully human, type $\mathrm{I}$, anti-CD20 $\mathrm{IgG}_{1} \kappa$ monoclonal antibody with a molecular weight of approximately $149 \mathrm{kDa} \cdot{ }^{1-6}$ Ofatumumab induces killing of tumor B-cell lines and primary tumor cells via activation of complement-dependent cytotoxicity (CDC) and antibody-dependent cell-mediated cytotoxicity (ADCC) in vitro. ${ }^{7,8}$ Compared with rituximab, which is a chimeric anti-CD20 antibody that has been in use for more than 15 years, ofatumumab has similar ADCC but increased binding of $\mathrm{C1q}$ and stronger CDC, as well as a slower off-rate and stabler CD20 binding. ${ }^{2,8,9}$ It also binds a different epitope of CD20 than rituximab. ${ }^{9}$ The more potent $\mathrm{CDC}$ with ofatumumab could be the result of the greater proximity of its CD20-binding site to the cell membrane, potentially leading to more effective deposition of complement on the cell surface..$^{7-10}$

Another anti-CD20 antibody that was recently approved by the US Food and Drug Administration (FDA) and European Medicines Agency (EMA) is obinutuzumab (also known as GA101), a humanized, glycoengineered type 2 antibody that targets a different CD20 epitope than ofatumumab. ${ }^{11}$ In preclinical studies, obinutuzumab was superior to rituximab in inducing ADCC, but was less effective with respect to CDC. ${ }^{12-16}$
Correspondence: Luca Laurenti Department of Hematology, Catholic University of the Sacred Heart, 8 Largo Gemelli, Rome I-00168, Italy

Tel +390630156016

Fax +3906 301 7319

Email I.laurenti@rm.unicatt.it 


\section{Ofatumumab as a single agent in relapsed/refractory CLL}

The safety and efficacy of ofatumumab monotherapy was initially evaluated in a Phase I/II dose-escalation trial comprising 33 heavily pretreated patients with relapsed/ refractory chronic lymphocytic leukemia (CLL; Table 1). ${ }^{1}$ Ofatumumab was well tolerated, with almost all adverse events (AEs) being grade $1 / 2$, displaying a similar toxicity as what would be expected in the same patient population with rituximab: $56 \%$ of AEs were infusion-related, and these were usually reduced in number and severity with subsequent infusions; and $51 \%$ of patients experienced infections and $15 \%$ had hematologic toxicity. The overall response rate (ORR) was $50 \%$. Time to response was rapid, with $62 \%$ of patients responding within 4 weeks.

Based on these promising results, a pivotal Phase II trial of ofatumumab monotherapy was conducted in patients with fludarabine- and alemtuzumab-refractory (FA-ref) CLL and in patients with bulky lymphadenopathy refractory to fludarabine (BF-ref) not suitable for treatment with alemtuzumab. ${ }^{3}$ Patients received eight weekly infusions of ofatumumab followed by four monthly infusions during a 24 -week period (dose 1, $300 \mathrm{mg}$; doses 2-12, 2,000 mg). Response was assessed every 4 weeks until week 24 , and then every 3 months until month 24 . The results from a planned interim analysis that included 138 treated patients with FA-ref $(\mathrm{n}=59)$ or BFref $(\mathrm{n}=79)$ CLL were reported by Wierda et al. ${ }^{3}$ ORRs were $58 \%$ and $47 \%$ in the FA-ref and BF-ref groups, respectively. Median progression-free survival (PFS) and overall survival (OS) times were 5.7 and 13.7 months in the FA-ref group, respectively, and 5.9 and 15.4 months in the BF-ref group, respectively. The most common AEs during treatment were infusion reactions and infections, which were primarily grade 1 or 2 events. Hematologic AEs during treatment included anemia and neutropenia. On the basis of the positive data from this trial, ${ }^{3}$ the FDA approved the use of ofatumumab in patients with FA-ref CLL in 2009,,$^{17,18}$ and the EMA granted conditional approval for the same indication in $2010 .{ }^{19}$
To gain a potential insight into the outcome of patients previously treated with or refractory to rituximab, Wierda et al subsequently performed an ad hoc retrospective analysis of the final 96 FA-ref and 111 BF-ref patients. ${ }^{20}$ This comprised 117 patients previously treated with rituximab, among which 98 were rituximab-refractory, and 89 patients that were rituximab-naïve. For rituximab-treated, rituximab-refractory, and rituximab-naïve patients, ORR was $43 \%, 44 \%$, and $53 \%$, median PFS was 5.3, 5.5, and 5.6 months, and median OS was $15.5,15.5$, and 20.2 months, respectively. No significant differences were observed in ofatumumab-related infusion reactions or hematologic or infectious complications between the three subgroups. Overall, this study demonstrated that ofatumumab monotherapy is effective even in patients with rituximab-refractory disease, indicating that ofatumumab may have greater activity than rituximab in the setting of CLL. ${ }^{20}$

The final results from the pivotal ofatumumab-monotherapy study were recently reported by Österborg et al. ${ }^{11}$ The efficacy observed in the final analysis confirmed the results of the previous interim analysis. ${ }^{3}$ ORRs were not significantly different between patient groups by demographics, prior CLL therapy, disease characteristics, chromosomal abnormalities, CD38 status, or Fc-receptor polymorphism. The only exception were patients with $17 \mathrm{p}$ deletion, who had a lower RR (30\%) than patients without $17 p$ deletion (53\%). Similarly to the interim analysis, the final analysis showed that ofatumumab was well tolerated with no unexpected toxicities and no formation of antiofatumumab antibodies.

To evaluate the efficacy of ofatumumab retreatment and maintenance therapy, a subset of 29 patients from the pivotal ofatumumab-monotherapy study who had at least stable disease and then progressed were retreated with eight weekly ofatumumab infusions, followed by monthly infusions for up to 2 years. ${ }^{21}$ The ORR after 8 weeks of induction retreatment was $45 \%$, and disease control after 52 weeks of maintenance was reported in $24 \%$ of patients. Comparison of the results of the retreated patients with their initial results from the pivotal study showed longer response

Table I Clinical studies of single-agent ofatumumab in relapsed/refractory CLL

\begin{tabular}{|c|c|c|c|c|c|c|c|c|}
\hline Study & Phase & Treatment & \multicolumn{2}{|l|}{$\mathbf{n}$} & ORR (\%) & CR (\%) & Median PFS & Median OS \\
\hline Coiffier et al' & $\mathrm{I} / \mathrm{II}$ & Ofa & \multicolumn{2}{|c|}{27 (cohort C) } & 48 & 0 & 106 days & ND \\
\hline \multirow{2}{*}{ Wierda et $\mathrm{al}^{3}$} & ॥ & Ofa in FA-ref & 138 & 59 & 58 & 0 & 5.7 months & 13.7 months \\
\hline & & Ofa in BF-ref & & 79 & 47 & I & 5.9 months & I5.4 months \\
\hline Moreno et $\mathrm{al}^{22}$ & IV & Observational study & 103 & & 22 & 3 & 5 months & II months \\
\hline Byrd et $\mathrm{al}^{23}$ & III & Ibrutinib vs Ofa & 391 & & 43 vs 4 & 0 vs 0 & $\begin{array}{l}\text { Ibrutinib NR ( } 88 \% \text { at } \\
6 \text { months) vs } 8.1 \text { months' Ofa }\end{array}$ & $\begin{array}{l}\text { Both groups NR; at } \\
\text { I } 2 \text { months, } 90 \% \text { vs } 81 \%\end{array}$ \\
\hline
\end{tabular}

Abbreviations: CLL, chronic lymphocytic leukemia; ORR, overall response rate; CR, complete response; PFS, progression-free survival; OS, overall survival; Ofa, ofatumumab; FA-ref, fludarabine- and alemtuzumab-refractory; BF-ref, fludarabine-refractory (with bulky [ $>5 \mathrm{~cm}$ ] lymphadenopathy); ND, no data; NR, not reached. 
duration (24.1 vs 6.8 months) and longer time to next therapy (14.8 vs 12.3 months), whereas median PFS was similar (7.4 vs 7.9 months, respectively). These data suggest that ofatumumab retreatment and maintenance therapy is feasible in patients with heavily pretreated CLL, and may result in more durable disease control than initial ofatumumab treatment in a subset of patients.

Recently, the European Research Initiative on CLL published a large retrospective, Phase IV, noninterventional, observational study of ofatumumab therapy in heavily pretreated patients with poor-prognosis CLL. ${ }^{22}$ Notably, this study was not able to reproduce the ORR observed in the studies of Coiffier et $\mathrm{al}^{1}$ and Wierda et $\mathrm{al}^{3}{ }^{3}$ raising questions over the use of ofatumumab as monotherapy in relapsed/refractory CLL. The ORR based on an intention to treat was $22 \%$ (three complete response [CR], one incomplete CR, and 19 partial response), which was less than half that observed in the two pivotal trials upon which both FDA and EMA approval was obtained. Median PFS and OS (5 and 11 months, respectively) were also shorter than those reported by Wierda et al (6 and 14 months, respectively). ${ }^{3}$ The $\mathrm{AE}$ profile was comparable to that seen in the two previous trials, and included infusion-related reactions, cytopenias, and infections.

Ofatumumab was also recently compared to ibrutinib, the Bruton tyrosine-kinase inhibitor, in a randomized clinical trial of previously treated CLL patients. ${ }^{23}$ Median duration of PFS was 8.1 months in the ofatumumab group, whereas it was not reached in the ibrutinib group. OS at 12 months was $81 \%$ and $90 \%$ in the ofatumumab and ibrutinib groups, respectively. ORR was also significantly higher in the ibrutinib group (43\% vs 4\%, respectively). Both drugs were well tolerated, and grade 3 AEs occurred at similar frequency (neutropenia, 16\% ibrutinib vs 14\% ofatumumab; anemia, $5 \%$ ibrutinib vs $8 \%$ ofatumumab; pneumonia, $7 \%$ ibrutinib vs $5 \%$ ofatumumab). In light of the efficacy and safety data of ibrutinib, which has recently received FDA and EMA approval for use in previously treated CLL, the role of single-agent ofatumumab in the relapsed/refractory setting now appears questionable.

\section{Combination therapy with ofatumumab in relapsed/refractory CLL}

In view of the activity of ofatumumab as a single agent in patients with relapsed/refractory CLL, its use in combination with chemotherapy was subsequently evaluated (Table 2).

The Italian Group for Adult Hematological Diseases (GIMEMA) conducted a Phase II, noncomparative, openlabel, multicenter study to assess the efficacy and safety of ofatumumab in combination with bendamustine (BendOfa) in relapsed/refractory CLL. Patients received monthly intravenous infusions of ofatumumab (cycle 1, $300 \mathrm{mg}$ on day 1 and 1,000 $\mathrm{mg}$ on day 8; cycles 2-6: 1,000 $\mathrm{mg}$ on day 1 every 28 days) in combination with bendamustine administered at a dose of $70 \mathrm{mg} / \mathrm{m}^{2}$ on days 1 and 2, every 28 days for up to six cycles; 47 patients were evaluated. The ORR was $72.3 \%$ (95\% confidence interval [CI]: 57\%-84\%), with 17\% CRs. The OS and PFS after a median follow-up of 24.2 months were $83.6 \%$ (95\% CI: $73.0 \%-95.7 \%$ ) and $49.6 \%$ (95\% CI: $35.9 \%-68.6 \%$ ), respectively. Myelosuppression was the most common toxicity: grade 3-4 neutropenia was observed in $61.7 \%$ of patients, though grade $3-4$ infections were rare and occurred in only $6 \%$ of patients. The authors concluded that BendOfa is feasible and effective in relapsed/refractory CLL patients, including patients with high-risk clinical and

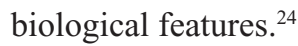

Offner et al reported in 2014 the preliminary results of a Phase II study with BendOfa therapy in patients with untreated or relapsed CLL. ${ }^{25}$ The primary outcome was ORR; secondary outcomes included complete RR, safety, and, tolerability. The ofatumumab schedule was the same as in

Table 2 Clinical studies of ofatumumab combination therapy in relapsed/refractory CLL

\begin{tabular}{|c|c|c|c|c|c|c|c|}
\hline Study & Phase & Treatment & $\mathbf{n}$ & ORR (\%) & CR (\%) & Median PFS & Median OS \\
\hline Cortelezzi et $\mathrm{al}^{24}$ & II & Ofa and Benda & 47 & 72 & 17 & 23.6 months & $83.6 \%$ at 24.2 months \\
\hline \multirow[t]{3}{*}{ Offner et $\mathrm{a}^{25}$} & II & Ofa and Benda in & 97 & & & NR & NR \\
\hline & & untreated and relapsed & 44 un & 95 un & 43 un & & \\
\hline & & & $53 \mathrm{rel}$ & 74 rel & II rel & & \\
\hline \multirow[t]{2}{*}{ Doubek et $\mathrm{a}^{26}$} & II & Ofa and dexamethasone & 33 & 67 & 15 & 10 months & 34 months \\
\hline & & & & $\operatorname{Del}(17 p) 63$ & Del (I7p) 25 & & \\
\hline \multirow[t]{2}{*}{ Costa et $\mathrm{a}^{29}$} & II & Sequential treatment with & 21 & 47.6 & ND & ND & 21.5 months \\
\hline & & Ofa and lenalidomide & & & & & \\
\hline Jaglowski et $\mathrm{al}^{30}$ & $\mid \mathrm{IB} / \mathrm{II}$ & Ofa and ibrutinib & 71 & 83.3 & 1.5 & 12 months & NR \\
\hline
\end{tabular}

Abbreviations: CLL, chronic lymphocytic leukemia; ORR, overall response rate; $C R$, complete response; PFS, progression-free survival; OS, overall survival; Ofa, ofatumumab; Benda, bendamustine; ND, no data; NR, not reached; Del, deleted; un, untreated; rel, relapsed. 
the study of GIMEMA. ${ }^{24}$ Patients without previous treatment received an initial bendamustine dose of $90 \mathrm{mg} / \mathrm{m}^{2}$. Patients with relapsed CLL received an initial dose of $70 \mathrm{mg} / \mathrm{m}^{2}$. The study enrolled a total of 97 patients: 44 patients with previously untreated CLL and 53 patients with relapsed CLL. The median ages of patients in the previously untreated and relapsed groups were 62.5 and 68.0 years, respectively. Patients in the relapsed group had received a median of one prior therapy, and $17 \mathrm{p}$ deletion was present in $12 \%$ of patients. Investigator-assessed ORRs were 95\% (CR $43 \%$ ) in the previously untreated population and $74 \%$ (CR $11 \%$ ) in the relapsed population. AEs classified as grade $\geq 3$ included neutropenia (previously untreated group, $n=16$; relapsed group, $n=29$ ), and thrombocytopenia (relapsed group, n=4). Four deaths occurred in the relapsed group, two of which were considered by the investigators to be possibly treatment-related. Grade $\geq 3$ infections occurred in $11 \%$ and $15 \%$ of previously untreated and relapsed patients, respectively. In the relapsed group, three patients had fatal infections. Grade $\geq 3$ infusion reactions occurred in $11 \%$ of previously untreated patients and $8 \%$ of relapsed patients. The authors concluded that BendOfa is an effective and tolerable therapy for both patients with relapsed CLL and patients with previously untreated CLL who are unfit for fludarabine-based therapy.

Doubek et al recently investigated the efficacy and toxicity of the combination of ofatumumab and high-dose dexamethasone in patients with relapsed/refractory CLL. ${ }^{26}$ The trial was a multicenter, nonrandomized, open-label, Phase II study. The ofatumumab-dexamethasone (O-Dex) regimen consisted of intravenous ofatumumab (cycle 1, $300 \mathrm{mg}$ on day $1,2,000 \mathrm{mg}$ on days 8,15 , and 22; cycles 2-6, 1,000 mg on days $1,8,15$, and 22) and oral dexamethasone (40 mg on days 1-4 and 15-18, cycles 1-6). The O-Dex regimen was given until best response or a maximum of six cycles. A total of 33 patients were recruited; 24 (73\%) completed at least three cycles of therapy, and nine (27\%) discontinued the protocol prematurely because of grade $3 / 4$ infections (seven patients), disease progression, or uncontrollable diabetes mellitus. The ORR and CR rate were $67 \%$ and $15 \%$, respectively. Median PFS was 10 months, and median OS was 34 months. In the eight patients with p53 mutation, ORR and CR rate were $63 \%$ and $25 \%$, whereas median PFS and OS were 10.5 and 34 months, respectively. The most frequent side effect during treatment were grade 3-5 infections, which occurred in $33 \%$ of patients. Overall, the results of this trial showed that O-Dex has considerable clinical activity in relapsed/refractory CLL patients, including patients with p53 abnormalities. Based on published data by the same group, O-Dex appears superior to the combination of rituximab and dexamethasone (R-Dex) in terms of PFS and OS (6 and 14.1 months, respectively, for R-Dex), whereas the ORR and CR rate $(62 \%$ and $21 \%$, respectively, for $\mathrm{R}-\mathrm{Dex})$ and frequency of serious infections (32\% for R-Dex) were similar. ${ }^{27}$

The results from the previous study are consistent with our own experience with ofatumumab and high-dose corticosteroids. ${ }^{28}$ In an earlier study, we treated three stage C/IV patients with refractory CLL that had received a median of four previous lines of therapy. All patients had grade 3 neutropenia, and one also had transfusion-dependent anemia and thrombocytopenia before treatment. The $17 \mathrm{p}$ deletion was present in two of the three patients. Patients received ofatumumab $(300 \mathrm{mg})$ on day 1 , followed by six doses of 2,000 $\mathrm{mg}$ from day 7 every 28 days, and high-dose methylprednisolone (HDMP; $1 \mathrm{~g} / \mathrm{m}^{2} /$ day), which was given intravenously for 5 days every 28 days for six consecutive cycles. Two patients responded with a partial response, whereas the third died from progressive disease after the third ofatumumab-HDMP cycle. This small experience further suggests that the ofatumumab-HDMP combination represents an effective and safe option for salvage therapy of heavily pretreated patients with severe cytopenia and a bad biological profile.

Ofatumumab has also been investigated in combination with lenalidomide. Costa et al recently published the results of a multicenter Phase II trial of sequential treatment with ofatumumab $(2,000 \mathrm{mg}$ intravenously on day 1$)$ and lenalidomide (10 mg on days 8-28) for up to six cycles in patients with advanced relapsed/refractory CLL. ${ }^{29}$ The rationale for the use of lenalidomide in association with ofatumumab derived from studies demonstrating that lenalidomide induces T-cell and natural killer-cell activation and enhances in vitro clearance of CLL cells by monoclonal antibodies. Twentyone subjects were included in the study, with a median age of 63 years and two prior lines of therapy. The ORR was $47.6 \%$, and median OS was 21.5 months. Neutropenia and thrombocytopenia were the most frequent AEs. A tumor-flare reaction occurred in $43 \%$ of subjects. The authors concluded that the combination of ofatumumab and lenalidomide is active in high-risk relapsed/refractory CLL and well tolerated, except for frequent cytopenias.

Recently, ofatumumab was evaluated in combination with ibrutinib, which induces a high RR as monotherapy, but produces few complete remissions. ${ }^{30,31}$ Patients with CLL/ small lymphocytic lymphoma, prolymphocytic leukemia, or Richter's transformation who had failed two or more prior 
therapies were enrolled. Because it was unknown whether the initial lymphocytosis commonly observed with ibrutinib would predispose to the development of ofatumumab infusion-related reactions or tumor lysis syndrome, three different administration schedules were evaluated. Patients received ibrutinib $420 \mathrm{mg}$ daily from 4 weeks before (group 1), 1 day after (group 2), or 8 weeks after (group 3) the start of ofatumumab treatment. Ofatumumab was administered for 12 doses: $300 \mathrm{mg}$ for dose 1, and 2,000 $\mathrm{mg}$ for the remaining eleven doses. A total of 71 patients were treated, and most had high-risk disease, including del17p13.1 in $44 \%$ or del11q22.3 in $31 \%$ of patients. The most frequent AEs were diarrhea (70\%), infusion-related reaction (45\%), peripheral sensory neuropathy (44\%), and stomatitis (38\%); these AEs were mostly grade $1 / 2$. The most common serious AEs were pneumonia $(16 \%)$, major bleeding events $(10 \%)$, and atrial fibrillation $(6 \%)$. There were no major differences between the three groups in the incidence of AEs or serious AEs.

The ORRs for the 66 CLL/small lymphocytic lymphoma patients belonging to groups 1,2 , and 3 were $100 \%, 79 \%$, and $71 \%$, respectively. Estimated 12 -month PFS for all patients were $89 \%, 85 \%$, and $75 \%$, respectively. Four patients in group 3 progressed prior to receiving ibrutinib. This study demonstrated that the combination of ibrutinib and ofatumumab is well tolerated and produces durable responses, with a quicker time to best response than single-agent ibrutinib.

\section{Ofatumumab as a single agent or in combinations for front-line therapy of CLL}

Following its approval for the treatment of patients with FA-ref CLL, ofatumumab was tested alone or in combination in untreated CLL patients (Table 3).

Flinn et al examined the role of ofatumumab monotherapy in the first-line setting in patients who were either $>65$ years old or younger who had declined fludarabine-based treatment. ${ }^{32}$ A total of 77 patients were enrolled, with a median age of
72 years. Ofatumumab was given weekly for 8 weeks, with an initial dose of $300 \mathrm{mg}$ followed by either $2,000 \mathrm{mg}$ (cohort 1) or $1,000 \mathrm{mg}$ (cohort 2). Maintenance therapy with ofatumumab (every 2 months) was also given over a period of 2 years to patients who responded to treatment or who did not have disease progression. The ORR for cohorts 1 and 2 was $55 \%$ and $36 \%$, with only two (5\%) and one (4\%) CR, respectively.

The low CR rate of ofatumumab as a single agent suggested that it should be combined with chemotherapy or B-cell receptor (BCR) inhibitors also in patients undergoing initial therapy. A randomized Phase II trial investigating ofatumumab plus fludarabine-cyclophosphamide (O-FC) in previously untreated patients was conducted by Wierda et al. ${ }^{33}$ A total of 61 patients received standard FC plus ofatumumab $300 \mathrm{mg}$ (first dose), followed by either $500 \mathrm{mg}$ ( $\operatorname{arm~A,~n=31)~}$ or 1,000 $\mathrm{mg}$ ( $\mathrm{arm} \mathrm{B}, \mathrm{n}=30$ ) monthly for the remaining five cycles. The ORR and CR rate were $77 \%$ and $32 \%$ in group A and $73 \%$ and $50 \%$ in group B, respectively. Although the ORR achieved with O-FC ${ }^{33}$ was lower than that reported with rituximab-FC, ${ }^{34-36}$ it should be noted that patients included in the ofatumumab trial had higher risk profiles $(13 \%$ had $17 \mathrm{p}$ deletion) than those included in the rituximab trials. The O-FC regimen was well tolerated, with the most common AEs being grade $1 / 2$ infusion-related reactions, mainly occurring during the first two infusions. Other common AEs were neutropenia (48\%), nausea (41\%), infections (38\%), thrombocytopenia (26\%), rash (25\%), vomiting (23\%), fever (21\%), headache $(18 \%)$, and fatigue $(18 \%)$. This trial demonstrated activity and feasibility of O-FC for the frontline treatment of patients with CLL, including high-risk patients.

Since many CLL patients were not suitable for fludarabine-based treatment because of old age and/or comorbidity, the combination of ofatumumab plus chlorambucil $(\mathrm{Clb})$ versus $\mathrm{Clb}$ monotherapy was evaluated in treatment-naïve patients by Hillmen et al in the COMPLEMENT-1 study. ${ }^{37}$ In this Phase III trial, 447 patients with a median age of 69 years

Table 3 Clinical studies with ofatumumab in untreated CLL patients

\begin{tabular}{|c|c|c|c|c|c|c|c|}
\hline Study & Phase & Treatment & $\mathbf{n}$ & ORR (\%) & CR (\%) & Median PFS & Median OS \\
\hline \multirow[t]{3}{*}{ Wierda et $\mathrm{a}^{33}$} & II & O-FC & 61 & & & NR & NR \\
\hline & & Ofa at different schedule in group $A$ & 31 pts group $A$ & 77 & 32 & & \\
\hline & & Group B & 30 pts group B & 73 & 50 & & \\
\hline \multirow[t]{3}{*}{ Hillmen et $\mathrm{al}^{37}$} & III & Ofa plus $\mathrm{Clb}$ vs $\mathrm{Clb}$ monotherapy & 447 & & & & NR at 29 months \\
\hline & & & 221 & 82 & 12 & 22.4 months & \\
\hline & & & 226 & 69 & I & 13.1 months & \\
\hline Shanafelt et al ${ }^{38}$ & ॥ & PCO & 48 & 96 & 46 & NR & NR \\
\hline
\end{tabular}

Abbreviations: CLL, chronic lymphocytic leukemia; ORR, overall response rate; $C R$, complete response; PFS, progression-free survival; OS, overall survival; Ofa, ofatumumab; O-FC, Ofa + fludarabine-cyclophosphamide; pts, patients; Clb, chlorambucil; PCO, pentostatin, cyclophosphamide, and ofatumumab; NR, not reached. 
were randomly assigned to $\mathrm{O}-\mathrm{Clb}$ (221 patients) or to $\mathrm{Clb}$ alone (226 patients). The ORR and CR rate for the $\mathrm{O}-\mathrm{Clb}$ arm were $82 \%$ and $12 \%$, respectively, compared to $69 \%$ and $1 \%$ with $\mathrm{Clb}$ alone. Median PFS was significantly prolonged with the addition of ofatumumab (22.4 vs 13.1 months). Median OS was not reached at a median follow-up of 29 months for either group. Grade $\geq 3$ AEs were more common in the $\mathrm{O}-\mathrm{Clb}$ than in the Clb-alone group ( $50 \%$ vs $43 \%$, respectively), with neutropenia being the most common event (26\% vs $14 \%$ ). However, the frequency of grade $\geq 3$ infections was similar in both groups. Based on these data, in 2014 the FDA and EMA approved the use of $\mathrm{O}-\mathrm{Clb}$ for first-line treatment of patients with CLL for whom fludarabine-based therapy is considered inappropriate.

Recently, the combination of obinutuzumab with $\mathrm{Clb}$ was also approved for the same group of patients. However, meaningful comparison between these two regimens is difficult, because of differences in study populations, study conduct, and $\mathrm{Clb}$ doses. ${ }^{37}$ Nevertheless, it is worth mentioning that the $\mathrm{O}-\mathrm{Clb}$ regimen currently has wider indications, because it can be prescribed to patients that are unsuitable for fludarabine-based regimens because of old age and/or comorbidity, whereas the obinutuzumab- $\mathrm{Clb}$ regimen is indicated only for the latter subgroup.

Ofatumumab has also been investigated in combination with pentostatin and cyclophosphamide (PCO) in previously untreated CLL patients. ${ }^{38}$ Patients in this trial received six cycles of induction therapy followed by response assessment. Of the 48 patients enrolled, 77\% completed PCO induction. The ORR was 96\% (46 of 48 patients) and the CR rate was $46 \%$ (22 of 48 patients). Among the 38 patients who underwent evaluation, seven (18\%) were negative for minimal residual disease. After a median follow-up of 24 months, ten (21\%) patients had progressed and eight (17\%) had required retreatment. AEs during induction included grade 3-4 hematologic toxicity in $27 \%$ and grade $3-4$ nonhematologic toxicity in $23 \%$ of patients. The efficacy and toxicity of PCO compared favorably to a historical trial of the same group using rituximab and an identical chemotherapy backbone. ${ }^{39}$ Time to retreatment also appeared longer for PCO than for the rituximabpentostatin-cyclophosphamide regimen (86\% vs $68 \%$ at 24 months, respectively). The authors concluded that PCO is well tolerated in patients with previously untreated CLL and that its efficacy compares favorably to historical trials of rituximab-pentostatin-cyclophosphamide, warranting the comparison of ofatumumab-based and rituximab-based chemoimmunotherapy regimens in randomized clinical trials.

\section{Ongoing studies with ofatumumab}

Several ongoing studies are investigating single-agent ofatumumab as maintenance therapy or combinations of ofatumumab with chemotherapy or BCR-pathway inhibitors in various settings (Table 4).

van Oers et al were exploring in a Phase III, randomized, multicenter trial the benefit of maintenance treatment in patients with relapsed CLL following a response to second- or third-line therapy (PROLONG).${ }^{40}$ Patients were randomized 1:1 to either intravenous ofatumumab $(300 \mathrm{mg}$ followed 1 week later by $1,000 \mathrm{mg}$ every 8 weeks for up to 2 years) or observation. A total of 474 patients randomized prior to the interim analysis were assessed. After a median follow-up of 26.1 months for the ofatumumab arm and 24 months for the observation arm, median PFS was 28.6 and 15.2 months, respectively (hazard ratio $[\mathrm{HR}]=0.48, P<0.0001)$. The median time to next treatment was 38 and 27.4 months in ofatumumab-treated patients and those in the observation arm, respectively (HR $=0.63, P=0.0076)$. At the time of the interim analysis, no significant difference in OS had been observed. Grade $3 / 4$ AEs were reported in $25 \%$ and $17 \%$ of patients in

Table 4 Ongoing studies with ofatumumab single agent or in combination in CLL

\begin{tabular}{|c|c|c|c|c|c|c|}
\hline Study & Phase & Treatment & $\mathbf{n}$ & ORR (\%) & $\begin{array}{l}\text { Median PFS, } \\
\text { months }\end{array}$ & Median OS, months \\
\hline van Oers et $\mathrm{al}^{40}$ & III & $\begin{array}{l}\text { Maintenance Ofa vs } \\
\text { observation in relapsed } \\
\text { patients after salvage therapy }\end{array}$ & 474 & $\begin{array}{l}\text { Time to next therapy } \\
38 \text { vs } 27.4 \text { months }\end{array}$ & 28.6 vs 15.2 & $\begin{array}{l}\text { No differences in OS } \\
\text { at the interim analysis }\end{array}$ \\
\hline Robak et $\mathrm{al}^{41}$ & III & $\begin{array}{l}\text { Ofa plus FC vs FC alone in } \\
\text { relapsed patients }\end{array}$ & $\begin{array}{l}365 \\
183 \text { vs } 182\end{array}$ & 84 vs 68 & 28.9 vs 18.8 & 56.4 vs 45.8 \\
\hline Jones et $\mathrm{al}^{42}$ & III & $\begin{array}{l}\text { Ofa plus Idela vs Ofa in } \\
\text { relapsed patients }\end{array}$ & $\begin{array}{l}26 \mid \\
\mid 74 \text { vs } 87\end{array}$ & 75 vs 18 & 16.3 vs 8.0 & 20.9 vs 19.4 \\
\hline
\end{tabular}

Abbreviations: CLL, chronic lymphocytic leukemia; ORR, overall response rate; PFS, progression-free survival; OS, overall survival; Ofa, ofatumumab; FC, fludarabine + cyclophosphamide; Idela, idelalisib. 
the ofatumumab and observation arms, respectively, and included neutropenia ( $22 \%$ ofatumumab vs $9 \%$ observation), infection (18\% ofatumumab vs $13 \%$ observation), and pneumonia ( $7 \%$ ofatumumab vs $4 \%$ observation). Based on these data, an application to expand the label to use ofatumumab as a maintenance therapy for patients with relapsed CLL was submitted earlier this year to the EMA and FDA.

Another ongoing Phase III study is evaluating O-FC vs FC alone in patients with relapsed CLL (COMPLEMENT 2). ${ }^{41}$ A total of 365 patients were randomized to receive either ofatumumab (300 $\mathrm{mg}$ on day 1 and 1,000 $\mathrm{mg}$ on day 8 in cycle 1 , followed by $1,000 \mathrm{mg}$ on day 1 in the subsequent five cycles) plus fludarabine ( $25 \mathrm{mg} / \mathrm{m}^{2}$ intravenously) and cyclophosphamide ( $250 \mathrm{mg} / \mathrm{m}^{2}$ intravenously) on days $1-3$ every 28 days for six cycles or only FC at the same schedule every 28 days for six cycles. The primary objective was to compare the PFS between the two treatments; secondary objectives were ORR, OS, safety, tolerability, and pharmacokinetics of $\mathrm{O}-\mathrm{FC}$ treatment versus FC therapy. Median PFS in patients receiving $\mathrm{O}-\mathrm{FC}$ was 28.9 months compared with 18.8 months in the $\mathrm{FC}$-alone $\operatorname{arm}(P=0.0032)$; median OS was 56.4 months in patients receiving $\mathrm{O}-\mathrm{FC}$ and 45.8 months in patients receiving only $\mathrm{FC}(P=0.1410)$. The ORR was significantly higher in the O-FC arm compared with the control FC arm (84\% and $68 \%$, respectively; $P=0.0003$ ). AEs were experienced in $93 \%$ of patients in the O-FC group compared with $85 \%$ of patients in the FC-only arm; a higher incidence of grade $\geq 3$ neutropenia was observed in the O-FC arm (58\%) compared with the FC-only arm (41\%), but this did not result in a substantially higher rate of infections. The results of this randomized study demonstrate that O-FC has improved efficacy compared to FC alone and that this combination has a manageable sideeffect profile in patients with previously treated CLL. ${ }^{41}$

Recently idelalisib, an oral PI3K inhibitor, was approved for previously treated CLL in combination with rituximab in patients for whom rituximab alone would be considered appropriate therapy due to other comorbidities. An openlabel Phase III study is evaluating the efficacy of combining ofatumumab and idelalisib in patients with previously treated CLL. ${ }^{42}$ Fit or unfit patients with CLL that had progressed $<24$ months from completion of last therapy were included in the study and randomized to receive idelalisib plus ofatumumab vs ofatumumab alone. Patients received idelalisib $150 \mathrm{mg}$ twice daily continuously and ofatumumab $300 \mathrm{mg}$ on day 1 of week 1 and then $1,000 \mathrm{mg}$ on day 1 of weeks $2-8$, followed by monthly infusions for 4 months (total 12 doses). In the control group, ofatumumab was administered at $300 \mathrm{mg}$ in week 1, 2,000 $\mathrm{mg}$ in weeks 2-8, and then monthly during the next 4 months. A total of 261 patients were included in the study: 174 received idelalisib plus ofatumumab, and 87 ofatumumab alone. The median age was 68 years, $40 \%$ of patients had del17p and/or p53 mutation, and $47 \%$ were refractory to prior therapy. ORR was $75.3 \%$ for patients receiving idelalisib/ ofatumumab and $18.4 \%$ for patients receiving ofatumumab alone $(P<0.0001)$. Median PFS was 16.3 and 8 months for patients receiving idelalisib/ofatumumab and ofatumumab alone, respectively $(P<0.0001)$, whereas median OS was not significantly different (20.9 vs 19.4 months). In the del17p/TP53 subgroup, the median PFS for the idelalisib + ofatumumab group was 13.7 months vs 5.8 for ofatumumab alone $(\mathrm{HR}=0.32,95 \% \mathrm{CI}: 0.18-0.57 ; P<0.0001)$. These data demonstrate that the idelalisib-ofatumumab combination yields superior PFS and ORR compared to ofatumumab alone in relapsed CLL, including within high-risk subgroups.

\section{Conclusion}

Ofatumumab in combination with $\mathrm{Clb}$ or bendamustine is approved for first-line treatment of patients with CLL for whom fludarabine-based therapy is considered inappropriate. For older patients with comorbidities who may not be able to tolerate intensive chemoimmunotherapy, these combinations appear an attractive option. Ofatumumab also has EMA and FDA authorization for the treatment of patients with CLL refractory to fludarabine and alemtuzumab, but its use in this setting appears questionable, given the availability of other more effective therapeutic options. The same refers to O-Dex, which demonstrated considerable activity in patients with relapsed/refractory CLL, including patients with p53 mutations, but is considerably more toxic than other more active novel options, such as ibrutinib or idelalisib with rituximab.

A possible novel indication for ofatumumab could be as maintenance therapy in patients with relapsed CLL who respond to induction therapy; an application to expand the label to use ofatumumab in this setting was recently submitted to the EMA and FDA based on the impressive results of the PROLONG study. Finally, combinations with BCRpathway inhibitors, such as ibrutinib and idelalisib, may represent another setting for ofatumumab in the future, given the promising activity of these combinations in patients with relapsed/refractory CLL.

\section{Disclosure}

The authors report no conflicts of interest in this work. 


\section{References}

1. Coiffier B, Lepretre S, Pedersen LM, et al. Safety and efficacy ofatumumab, a fully human monoclonal anti-CD20 antibody, in patients with relapsed or refractory B-cell chronic lymphocytic leukemia: a phase 1-2 study. Blood. 2008;111:1094-1100.

2. Robak T. Ofatumumab, a human monoclonal antibody for lymphoid malignancies and autoimmune disorders. Curr Opin Mol Ther. 2008; 10:294-309.

3. Wierda WG, Kipps TJ, Mayer J, et al. Ofatumumab as single-agent CD20 immunotherapy in fludarabine-refractory chronic lymphocytic leukemia. J Clin Oncol. 2010;28:1749-1755.

4. Sanford M, McCormack PL. Ofatumumab. Drugs. 2010;70:1013-1019.

5. Lemery SJ, Zhang J, Rothmann MD, et al. U.S. Food and Drug Administration approval: ofatumumab for the treatment of patients with chronic lymphocytic leukemia refractory to fludarabine and alemtuzumab. Clin Cancer Res. 2010;16:4331-4338.

6. Arzerra (ofatumumab) [prescribing information]. Research Triangle Park (NC): GlaxoSmithKline; 2009.

7. Beum PV, Lindorfer MA, Beurskens F, et al. Complement activation on B lymphocytes opsonized with rituximab or ofatumumab produces substantial changes in membrane structure preceding cell lysis. J Immunol. 2008;181:822-832.

8. Teeling JL, French RR, Cragg MS, et al. Characterization of new human CD20 monoclonal antibodies with potent cytolytic activity against non-Hodgkin lymphomas. Blood. 2004;104:1793-1800.

9. Teeling JL, Mackus WJ, Wiegman LJ, et al. The biological activity of human CD20 monoclonal antibodies is linked to unique epitopes on CD20. J Immunol. 2006;177:362-371.

10. Pawluczkowycz A, Beurskens F, Beum PV, et al. Binding of submaximal C1q promotes complement dependent cytotoxicity (CDC) of B cells opsonized with anti-CD20 mAbs ofatumumab (OFA) or rituximab (RTX): considerably higher levels of CDC are induced by OFA than by RTX. J Immunol. 2009;183:749-758.

11. Österborg A, Jewell RC, Padmanabhan-Iyer S, et al. Ofatumumab monotherapy in fludarabine-refractory chronic lymphocytic leukemia: final results from a pivotal study. Haematologica. 2015;100: e311-e314.

12. Mössner E, Brünker $P$, Moser $S$, et al. Increasing the efficacy of CD20 antibody therapy through the engineering of a new type II anti-CD20 antibody with enhanced direct and immune effector cell-mediated B-cell cytotoxicity. Blood. 2010;115:4393-4402.

13. Patz M, Isaeva P, Forcob N, et al. Comparison of the in vitro effects of the anti-CD20 antibodies rituximab and GA101 on chronic lymphocytic leukaemia cells. Br J Haematol. 2011;152:295-306.

14. Dalle S, Reslan L, Besseyre de Horts T, et al. Preclinical studies on the mechanism of action and the anti-lymphoma activity of the novel anti-CD20 antibody GA101. Mol Cancer Ther. 2011;10:178-185.

15. Alduaij W, Ivanov A, Honeychurch J, et al. Novel type II anti-CD20 monoclonal antibody (GA101) evokes homotypic adhesion and actin-dependent, lysosome-mediated cell death in B-cell malignancies. Blood. 2011;117:4519-4529.

16. Herter S, Herting F, Mundigl O, et al. Preclinical activity of the type II CD20 antibody GA101 (obinutuzumab) compared with rituximab and ofatumumab in vitro and in xenograft models. Mol Cancer Ther. 2013;12:2031-2042.

17. GlaxoSmithKline. Arzerra (ofatumumab) [prescribing information]. Available from: www.accessdata.fda.gov/drugsatfda_docs/ label/2009/125326lbl.pdf. Accessed February 25, 2012.

18. Reuters. GSK and Genmab receive accelerated approval for Arzerra. 2009. Available from: http://www.reuters.com/article/2009/10/26/ idUS228795+26-Oct-2009+GNW20091026\#uQUJ9XbcxYWgmY20.97. Accessed December 3, 2015.

19. FirstWord Pharma. GlaxoSmithKline receives conditional marketing authorization in the EU for Arzerra (ofatumumab). 2010. Available from: http://www.firstwordpharma.com/node/606884\#axzz3tCYbaRcM. Accessed December 3, 2015.
20. Wierda WG, Padmanabhan S, Chan GW, Gupta IV, Lisby S, Österborg A. Ofatumumab is active in patients with fludarabinerefractory CLL irrespective of prior rituximab: results from the phase 2 international study. Blood. 2011;118:5126-5129.

21. Österborg A, Wierda WG, Mayer J, et al. Ofatumumab retreatment and maintenance in fludarabine refractory chronic lymphocytic leukaemia patients. Br J Haematol. 2015;170:40-49.

22. Moreno C, Montillo M, Panayotidis $\mathrm{P}$, et al. Ofatumumab in poor-prognosis chronic lymphocytic leukemia: a phase IV, non-interventional, observational study from the European Research Initiative on Chronic Lymphocytic Leukemia. Haematologica. 2015;100:511-516.

23. Byrd JC, Brown JR, O'Brien S, et al. Ibrutinib versus ofatumumab in previously treated chronic lymphoid leukemia. N Engl J Med. 2014; 371:213-223.

24. Cortelezzi A, Sciumè M, Liberati AM, et al. Bendamustine in combination with ofatumumab in relapsed or refractory chronic lymphocytic leukemia: a GIMEMA multicenter phase II trial. Leukemia. 2014;28:642-648.

25. Offner F, Panagiotidis P, Afanasyev B, et al. Ofatumumab and bendamustine combination therapy in patients with untreated and relapsed chronic lymphocytic leukemia: initial results of the phase II study OMB115991. 2014. Available from: http://www.newevidence. com/oncology/ofatumumab-and-bendamustine-combination-therapyin-patients-with-untreated-and-relapsed-chronic-lymphocyticleukemia-initial-results-of-the-phase-ii-study-omb115991. Accessed December 3, 2015.

26. Doubek M, Brychtova Y, Panovska A, et al. Ofatumumab added to dexamethasone in patients with relapsed or refractory chronic lymphocytic leukemia: results from a phase II study. Am J Hematol. 2015;90: 417-421.

27. Smolej L, Doubek M, Panovská A, et al. Rituximab in combination with high-dose dexamethasone for the treatment of relapsed/refractory chronic lymphocytic leukemia. Leuk Res. 2012;36:1278-1282.

28. Vannata B, Innocenti I, Autore F, et al. High-dose glucocorticoids plus ofatumumab in fludarabine/alemtuzumab-resistant B-cell chronic lymphocytic leukemia. Am J Hematol. 2012;87:E133.

29. Costa LJ, Fanning SR, Stephenson J, et al. Sequential ofatumumab and lenalidomide for the treatment of relapsed and refractory chronic lymphocytic leukemia and small lymphocytic lymphoma. Leuk Lymphoma. 2015;56:645-649.

30. Jaglowski SM, Jones JA, Nagar V, et al. Safety and activity of BTK inhibitor ibrutinib combined with ofatumumab in chronic lymphocytic leukemia: a phase 1B/2 study. Blood. 2015;126:842-850.

31. Byrd JC, Furman RR, Coutre SE, et al. Three-year follow-up of treatment-naïve and previously treated patients with CLL and SLL receiving single-agent ibrutinib. Blood. 2015;125:2497-2506.

32. Flinn IW, Harwin WN, Ward P, et al. Phase II trial of ofatumumab (OFA) for older patients and patients who refuse fludarabine-based regimens with previously untreated chronic lymphocytic leukemia (CLL) or small lymphocytic lymphoma (SLL). Poster presented at: 54th ASH Annual Meeting and Exposition; December 8-11, 2012; Atlanta, GA.

33. Wierda WG, Kipps TJ, Dürig J, et al. Chemoimmunotherapy with O-FC in previously untreated patients with chronic lymphocytic leukemia. Blood. 2011;117:6450-6458.

34. Hallek M, Fischer K, Fingerle-Rowson G, et al. Addition of rituximab to fludarabine and cyclophosphamide in patients with chronic lymphocytic leukaemia: a randomised, open-label, phase 3 trial. Lancet. 2010;376: 1164-1174.

35. Keating MJ, O'Brien S, Albitar M, et al. Early results of a chemoimmunotherapy regimen of fludarabine, cyclophosphamide, and rituximab as initial therapy for chronic lymphocytic leukemia. J Clin Oncol. 2005; 23:4079-4088.

36. Tam CS, O'Brien S, Wierda WG, et al. Long-term results of the fludarabine, cyclophosphamide, and rituximab regimen as initial therapy of chronic lymphocytic leukemia. Blood. 2008;112:975-980. 
37. Hillmen P, Robak T, Janssens A, et al. Chlorambucil plus ofatumumab versus chlorambucil alone in previously untreated patients with chronic lymphocytic leukaemia (COMPLEMENT 1): a randomised, multicentre, open-label phase 3 trial. Lancet. 2015;385:1873-1883.

38. Shanafelt T, Lanasa MC, Call TG, et al. Ofatumumab-based chemoimmunotherapy is effective and well tolerated in patients with previously untreated chronic lymphocytic leukemia (CLL). Cancer. 2013;119: 3788-3796.

39. Kay NE, Geyer SM, Call TG, et al. Combination chemoimmunotherapy with pentostatin, cyclophosphamide, and rituximab shows significant clinical activity with low accompanying toxicity in previously untreated B chronic lymphocytic leukemia. Blood. 2007;109:405-411.

40. van Oers MH, Kuliczkowski K, Smolej L, et al. Ofatumumab maintenance versus observation in relapsed chronic lymphocytic leukaemia (PROLONG): an open-label, multicentre, randomised phase 3 study. Lancet Oncol. 2015;16:1370-1379.
41. Robak T, Grosicki S, Warzocha K, et al. Ofatumumab $(\mathrm{O})$ in combination with fludarabine (F) and cyclophosphamide (C) (OFC) vs FC in patients with relapsed chronic lymphocytic leukemia (CLL): results of the phase III study COMPLEMENT 2. Poster presented at: 20th Congress of European Hematology Association; June 11-14, 2015; Vienna, Austria.

42. Jones J, Wach M, Robak T, et al. Results of a phase III randomized, controlled study evaluating the efficacy and safety of idelalisib (IDELA) in combination with ofatumumab (OFA) for previously treated chronic lymphocytic leukemia (CLL). J Clin Oncol. 2015;33 Suppl:7023.

\section{Publish your work in this journal}

OncoTargets and Therapy is an international, peer-reviewed, open access journal focusing on the pathological basis of all cancers, potential targets for therapy and treatment protocols employed to improve the management of cancer patients. The journal also focuses on the impact of management programs and new therapeutic agents and protocols on

\section{Dovepress}

patient perspectives such as quality of life, adherence and satisfaction. The manuscript management system is completely online and includes a very quick and fair peer-review system, which is all easy to use. Visit http://www.dovepress.com/testimonials.php to read real quotes from published authors.

Submit your manuscript here: http://www.dovepress.com/oncotargets-and-therapy-journal 